The Astrophysical Journal, 412:L43-L46, 1993 July 20

(C) 1993. The American Astronomical Society. All rights reserved. Printed in U.S.A.

\title{
SUBARCSECOND IMAGES OF CW TAURI: CONSTRAINTS ON DISK SIZE AND JET FORMATION
}

\author{
ANA I. GómEZ DE CASTRO ${ }^{1,2}$ \\ Received 1992 December 23; accepted 1993 April 22
}

\begin{abstract}
Subarcsecond spatial resolution images in the $[\mathrm{S}$ II $]$ and $[\mathrm{N}$ II $]+\mathrm{H}_{\alpha}$ emission lines of the $\mathrm{T}$ Tauri star (TTS) CW Tau are presented. It is shown that CW Tau has a jet that extends up to 2 "2 (300 AU) from the star in the direction P.A. $=144^{\circ}$. The jet emits strongly in [S II], and it is already collimated at $1^{\prime \prime} 3(176 \mathrm{AU})$ from the star.

The images also indicate that $\mathrm{CW}$ Tau is extended in the east-west direction and that the jet is slightly shifted to the east. This suggests that $\mathrm{CW}$ Tau may be a binary with the two components separated 0 ".20 (28 AU). The observations are compared with the theoretical models proposed for the production of forbidden line emission regions (FLERs) and protostellar jets.
\end{abstract}

Subject headings: stars: formation - stars: individual (CW Tauri, FM Tauri, V773 Tauri) stars: pre-main-sequence

\section{INTRODUCTION}

Some T Tauri stars (TTSs) have strong forbidden line emission in [S II], [O I ], and [N II] coming from spatial scales $\leq 100 \mathrm{AU}$. These lines have some intriguing properties. They seem to be excited by shocks (Hartmann \& Raymond 1988, hereafter $\mathrm{HL})$ and are stronger $\left(W\left(\left[\mathrm{O}_{\mathrm{I}}\right]\right) \geq 1 \AA\right)$ in the TTSs associated with optical jets and bipolar outflows (Gómez de Castro \& Pudritz 1993, hereafter GP). This suggests that the formation of optical jets and the detection of forbidden line emission regions (FLERs) are related phenomena. Moreover, the forbidden line strength is correlated with the infrared excess. Since the infrared excess seems to be produced by the thermal emission of protostellar accretion disks, this correlation has been interpreted as a proof of the connection between jets and accretion disks (Cabrit et al. 1990). Highresolution imaging of FLERs provides a first-hand observational test of the physical models proposed for their generation (HL; GP) and its possible relation with jets and accretion. Subarcsecond images of the TTS CW Tau are presented in this Letter.

\section{OBSERVATIONS}

The observations were carried out in 1991 February with the high-resolution camera (HRC) of the Canada-France-Hawaii Telescope (CFHT). The detector used was a SAIC 2 CCD which has a field of view of $2.2 \times 2.2$ and a pixel size of $18 \mu \mathrm{m}$. The scale is 0.13 pixel $^{-1}$.

The images have spatial resolutions of $0.5-0$ ".8 (70-112 AU at Taurus distance $\simeq 140 \mathrm{pc}$ ) and were obtained in three narrow-band filters, namely, $\left[\begin{array}{ll}\mathrm{O} & \mathrm{I}\end{array}\right]_{5577},\left[\begin{array}{ll}\mathrm{S} & \mathrm{II}\end{array}\right]_{6729}$, and $[\mathrm{N} \mathrm{II}]_{6584}$, and an intermediate-band filter centered at $6100 \AA$ $\left(\lambda_{c}=6100 \AA\right.$ and FWHM $\left.=198 \AA\right)$. The specifications of the filters are described in Gómez de Castro \& Pudritz (1992). The data were reduced using the IRAF and MIDAS image pro-

\footnotetext{
${ }^{1}$ IUE Observatory-VILSPA (affiliated to the Astrophysics Division, Space Science Department, ESTEC), P.O. Box 50727, 28080 Madrid, Spain.

2 Visiting Astronomer, Canada-France-Hawaii Telescope, which is operated by the National Research Council of Canada, the Centre National de la Recherche Scientifique de France, and the University of Hawaii.
}

cessing packages. Images were bias-corrected and flat-fielded in the standard manner.

There are three $\mathrm{T}$ Tauri stars in the field: CW Tau (our primary target), FM Tau, and V773 Tau. There is also a $V \simeq 18$ mag field star. V773 Tau was used as the guide star for the fast tracking of the camera (see McClure et al. 1989 for details on HRC). Images with similar good seeing have been co-added to obtain the final high signal-to-noise ratio frames shown in Figure 1. A two-dimensional Gaussian fitting has been applied to all the sources in the images, and the resulting FWHMs in the $\alpha$ and $\delta$ directions are given in Table 1 . A reliable fitting to the field star can only be made in the coadded frames. The field star is the most reliable tracer of the point-spread function (PSF) that is typically $9 \% \pm 3 \%$ wider in $\alpha$ than in $\delta$ (see Table 1). FM Tau is extended and similar in all the frames, indicating that there is a small-scale reflection nebula around the star.

\section{RESULTS}

\subsection{CW Tau Is Not Pointlike}

CW Tau's FWHM is typically $25 \% \pm 3 \%$ wider in $\alpha$ than in $\delta$, and therefore it is more elongated than the rest of the stars. Variations of the PSF across the CCD have been reported in some high-quality images obtained with CFHT + HRC (McClure et al. 1991). These are due to isoplanatism anisotropies and significantly $(\sim 12 \%)$ distort the PSF in the direction toward the guide star at large distances ( $\sim 700$ pixels) from the guide. This anisotropy would elongate $\mathrm{CW}$ Tau's image in the direction P.A. $=-43^{\circ}$ (toward V773 Tau) and not toward P.A. $=90^{\circ}$ as observed. Therefore, the reported variations of the PSF cannot explain the morphology of CW Tau, and, hence, one may conclude that CW Tau is an extended source.

The morphology of both CW Tau and the PSF can be modeled by a two-dimensional Gaussian with the residuals $\leq 8 \%$. Therefore, the underlying shape of the source, found after deconvolution with the PSF, may also be described by a two-dimensional Gaussian with FWHM in $\alpha$ and $\delta$ directions $\simeq 0$ ".43 and $\simeq 0$ ". 22 , respectively (see Table 1 ). This indicates that CW Tau is at least twice as large in $\alpha$ as in $\delta$. 
[N II $] 6584$

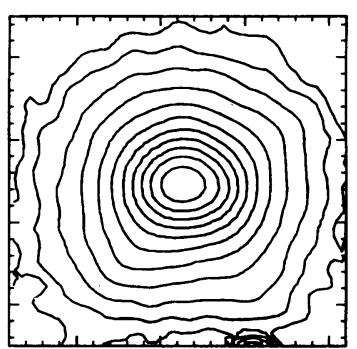

[S II $] 6730$

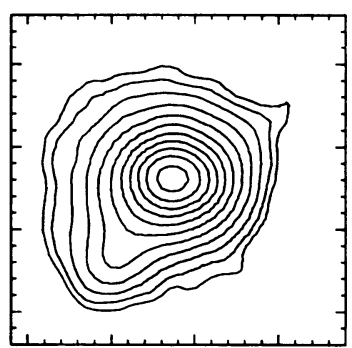

[O 1 I $] 5577$

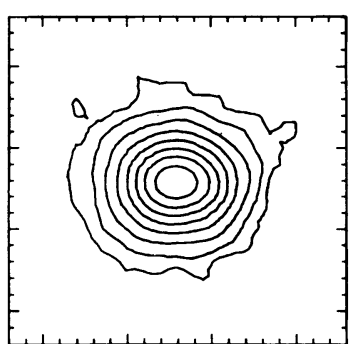

$C-610$

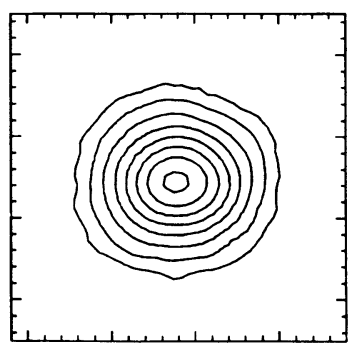

FIG. 1.-Contour plots of $\mathrm{CW}$ Tau in the $[\mathrm{N} \mathrm{III}]_{6584},[\mathrm{~S} \mathrm{II}]_{6730},[\mathrm{O} \mathrm{I}]_{5577}$, and continuum (C610) bands. The levels plotted in each panel are the same and correspond to the logarithmic scale: $2 \sigma, 4 \sigma, 8 \sigma, 16 \sigma, 32 \sigma, 64 \sigma, 128 \sigma, 256 \sigma$, $512 \sigma, 1024 \sigma$, and $2048 \sigma$. The distance between two small tick marks is 0 ". 26 North is at the top, and east is to the left.

[S II] Restored

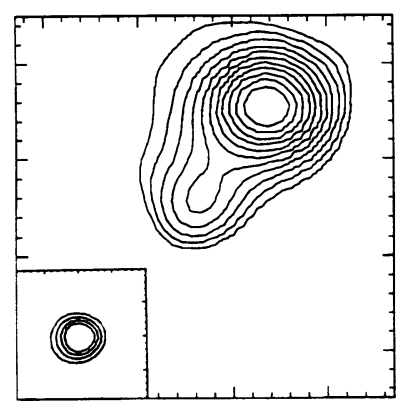

[NII] Restored

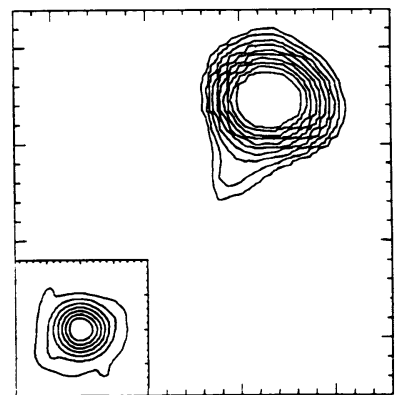

Fig. 2.-Restored [S II] (top) and [N II] (bottom) images after using the maximum-likelihood algorithm. The isocontour levels are the same as plotted in Fig. 1. The PSF is illustrated in the small panels for each filter. The distance between two small tick marks is 0 ". 26 in the small and in the big panels.

\subsection{The FLER Is Resolved into a Jet}

The $[\mathrm{S} \mathrm{II}]$ and $[\mathrm{N}$ II] images have been restored using the maximum-likelihood algorithm by Lucy (1974). The restored images and the PSF are shown in Figure 2. The number of iterations has been controlled by restoring the field star simultaneously. The restored [S II] image shows that there is a jet that extends $\sim 0$.9 $(122 \mathrm{AU})$ in the direction P.A. $=144^{\circ}$. The jet apparently starts in the bright condensation at $\simeq 1^{\prime \prime} .3(176$ AU) from $\mathrm{CW}$ Tau. The restored [N $\mathrm{NI}]$ frame shows $[\mathrm{N} \mathrm{II}]+\mathrm{H} \alpha$ (see below) emission coming from the brightest area of the jet, closer to the star.

\subsection{Extension of the FLERs: Net Flux in the Lines}

The morphology of the FLERs can be better studied by subtracting out the continuum from the narrow-band images. Since the expected line contribution to the total flux in the narrow-band filters is $\sim 2 \%$, the subtraction is very delicate. A rough estimate can be made using FM Tau for calibration. The spectra of FM Tau and CW Tau are known (Cohen \& Kuhi 1979, hereafter CK), and the color corrections can be calculated with an accuracy of $\sim 8 \%$. The ratio of CW Tau's to FM Tau's total flux in each one of the CCD images is given in Table 1. The color excess of CW Tau with respect to FM Tau in the $\left[\mathrm{O}_{\mathrm{I}}\right]-\mathrm{C} 610,[\mathrm{~S}$ II $]-\mathrm{C} 610$ and [N II]-C610 bands has been measured directly from the observations and compared with the expected values from CK published spectra. There is excellent agreement between the observed and the expected values, confirming that, indeed, the $\left[\mathrm{O}{ }_{1}\right]_{5577},[\mathrm{~S} \mathrm{II}]_{6731}$, and $[\mathrm{N} \mathrm{II}]_{6584}$ line contribution to the total flux in the [O I $]$, [S II], and $[\mathrm{N}$ II] images is smaller than $\simeq 8 \%$, respectively.

The net $[\mathrm{S}$ II $]$ and $[\mathrm{N}$ II $]$ emissions have been calculated from the nonrestored images after subtracting similar-seeing [O I] frames scaled with the values given in Table 1 . The [O I] images can be taken as "bona fide" continua and have better spatial resolution than the C610 frame (see Table 1). In addition, the following three criteria have been applied for the fine tuning of the net [S II] and [N II] emission images: (1) the jet has to be seen, (2) the stellar flux after subtraction ought to be always $\geq 0$, and (3) the total line-to-continuum flux ratio cannot be larger than that estimated from the spectra.

The $[\mathrm{S} \mathrm{II}]$ line emission is weak and concentrated in the jet. In the images shown in Figure 3 , the net flux in the jet is $1.0 \% \pm 0.3 \%$ of the stellar flux, which is in good agreement with the $\sim 1.6 \%$ expected from the spectra (Edwards et al. 1987). Long-slit high-resolution spectra show that approximately one-third of the line flux comes from the zero-velocity component that is centered in the star (Hirth, Mundt, \& Solf 1993). The distance from the beginning of the jet to the star is $176 \mathrm{AU}$, confirming the scale independently found from the restored images.

The net [N II] image in Figure 3 has been obtained using the color corrections given in Table 1 . The jet is barely seen and represents only $0.7 \% \pm 0.4 \%$ of the total flux detected in the [N II] filter. There is, however, significant emission from the star itself $(7.3 \% \pm 0.5 \%$ of the total). The emission represents a part of the $\mathrm{H} \alpha$ line flux that passes through the blue border of the [N II] filter. CW Tau's $\mathrm{H} \alpha$ emission line is very intense and broad, and the $[\mathrm{N}$ II] filter has $50 \%$ transmittance at $340 \mathrm{~km}$ $\mathrm{s}^{-1}$ from $\mathrm{H} \alpha$. Note that the dominant component of the $\mathrm{H} \alpha+[\mathrm{N}$ II $]$ emission extends perpendicular to the jet, approximately in the same direction as the reported elongation of $\mathrm{CW}$ Tau (see $\S 3.1$ ), and that the jet is shifted to the east. 
TABLE 1

SUMMARY OF MEASUREMENTS

\begin{tabular}{|c|c|c|c|c|c|c|c|c|c|c|}
\hline \multirow[b]{2}{*}{ FILTER } & \multirow{2}{*}{$\begin{array}{c}\text { AIR } \\
\text { MASS }\end{array}$} & \multicolumn{2}{|c|}{ CW TAU } & \multicolumn{2}{|c|}{ FM TAU } & \multicolumn{2}{|c|}{ FieLd STAR } & \multirow{2}{*}{$\begin{array}{c}R^{\mathrm{a}} \\
\text { Flux(CW Tau) } \\
\text { Flux(FM Tau) }\end{array}$} & \multicolumn{2}{|c|}{$R($ filter $) / R(\mathrm{C} 610)^{\mathrm{b}}$} \\
\hline & & $\mathrm{FWHM}_{\alpha}$ & $\mathrm{FWHM}_{\delta}$ & $\mathrm{FWHM}_{\alpha}$ & $\mathrm{FWHM}_{\delta}$ & $\mathrm{FWHM}_{\alpha}$ & $\mathrm{FWHM}_{\delta}$ & & Observed & CK \\
\hline$[\mathrm{S}$ II $]-1 \ldots \ldots$ & 1.179 & 0.64 & 0.53 & 0.68 & 0.67 & $\ldots$ & $\ldots$ & 1.572 & $\cdots$ & $\cdots$ \\
\hline$[\mathrm{S}$ II $]-2 \ldots \ldots$ & 1.202 & 0.62 & 0.51 & 0.67 & 0.64 & $\ldots$ & $\ldots$ & 1.555 & $\ldots$ & $\ldots$ \\
\hline$[\mathrm{S} \mathrm{II}]-3 \ldots \ldots$ & 1.228 & 0.62 & 0.49 & 0.67 & 0.62 & $\ldots$ & $\ldots$ & 1.545 & & $\ldots$ \\
\hline$\left[\mathrm{S}_{\mathrm{II}}\right]-$ tot $\ldots$. & $\cdots$ & 0.64 & 0.52 & 0.69 & 0.65 & 0.51 & 0.45 & 1.557 & 1.171 & $1.3 \pm 0.1$ \\
\hline 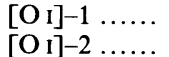 & $\begin{array}{l}1.255 \\
1.285\end{array}$ & $\begin{array}{l}0.67 \\
0.71\end{array}$ & $\begin{array}{l}0.52 \\
0.57\end{array}$ & 0.71 & 0.68 & $\cdots$ & $\ldots$ & $1.202^{\mathrm{c}}$ & $\cdots$ & $\cdots$ \\
\hline$\left[\begin{array}{lll}O_{I} & 1\end{array}\right]-3 \ldots \ldots$ & $\begin{array}{l}1.285 \\
1.361\end{array}$ & $\begin{array}{l}0.71 \\
0.68\end{array}$ & $\begin{array}{l}0.57 \\
0.53\end{array}$ & 0.78 & 0.71 & $\cdots$ & $\cdots$ & 1.136 & $\ldots$ & $\cdots$ \\
\hline$\left[\mathrm{O}_{\mathrm{I}}\right]$-tot .... & $\ldots$ & 0.70 & 0.55 & 0.76 & $\begin{array}{l}.00 \\
0.70\end{array}$ & 0.57 & 0.51 & $\begin{array}{l}1.137 \\
1.137\end{array}$ & 0.855 & $0.84+0.07$ \\
\hline$[\mathrm{N}$ II $]-1 \ldots \ldots$ & 1.462 & 0.70 & 0.56 & 0.76 & 0.68 & $\cdots$ & $\cdots$ & 1.408 & $\ldots$ & $\cdots$ \\
\hline$[\mathrm{N}$ II $]-2 \ldots$. & 1.585 & 0.75 & 0.59 & 0.79 & 0.69 & $\ldots$ & $\ldots$ & 1.420 & $\ldots$ & $\ldots$ \\
\hline$[\mathrm{N} \mathrm{II}]-3 \ldots \ldots$ & 1.683 & 0.75 & 0.59 & 0.80 & 0.71 & $\ldots$ & & 1.422 & & $\ldots$ \\
\hline$\left[\mathrm{N}_{\mathrm{II}}\right]$-tot $\ldots$ & $\ldots$ & 0.75 & 0.59 & 0.80 & 0.71 & 0.64 & 0.57 & 1.423 & 1.071 & $1.1 \pm 0.1$ \\
\hline C610 ........ & 1.870 & 0.78 & 0.65 & 0.82 & 0.74 & 0.67 & 0.64 & 1.328 & $\ldots$ & $\ldots$ \\
\hline
\end{tabular}

${ }^{a} R$ represents the ratio between the integrated flux of CW Tau and FM Tau in the same image. The flux has been integrated up to the radius where the intensity drops to $1 \%$ of the maximum.

${ }^{\mathrm{b}} R(\mathrm{filter}) / R(\mathrm{C} 610)$ represents the ratio between the values of $R$ measured in the [O $\left.\mathrm{I}\right]$, [S II], and [N II] filters and the value of $R$ measured in the $\mathrm{C} 610$ band, respectively. This ratio can be compared directly with the expected values from the Cohen \& Kuhi 1979 spectra.

${ }^{c}$ The flux of FM Tau has been integrated only up to $5 \%$ of the peak value, since this was the noise level in this particular frame.

\section{DISCUSSION AND CONCLUSIONS}

\subsection{Constraints on the Size of the Protostellar Disk/Envelope}

The elongated morphology of the continuum emission from CW Tau can be due either to the presence of a small-scale reflection nebula or to binarity. If it is produced by a reflection nebula, the images indicate that the dust is concentrated in a flattened structure with aspect ratio at least $1: 2$, that is, oriented approximately perpendicular to the outflow (see below for a discussion on binarity). If this structure can be described by a disk, its radius would be 0.22 (32 AU) and the scale height $0^{\prime \prime} .11$ (17 AU).

Moreover, since optical jets from young stars are often bipolar, the nondetection of the counterjet suggests that there is an envelope around the system extending up to radii $\geq 176$ AU. A lower limit to the extinction caused by the envelope can be estimated from the isocontours in Figure 3, indicating $A_{V} \geq 2$ mag. This value is similar to the extinction to CW Tau estimated from the optical spectra, $A_{V}=2.6 \mathrm{mag}$ (Beckwith et al. 1990, hereafter BSCG).

The inferred column density of the envelope around $\mathrm{CW}$ Tau is $\Sigma \geq(3.3-4) \times 10^{21} \mathrm{~cm}^{-2}$ (reddening laws $R=$ $A_{V} / E_{B-V}=3.2-6$; Scheffer 1982). This limit is equivalent to the column density of the protostellar cores mapped in $\mathrm{NH}_{3}$ by Benson \& Myers (1989) that have typical particle densities, $N \sim 10^{4} \mathrm{~cm}^{-3}$, and radius, $R \sim 0.1 \mathrm{pc}$.

\subsection{CW Tau: A Possible Binary}

CW Tau is extended in a direction $\sim 54^{\circ}$ from the jet axis, and the jet axis seems to be shifted to the east side of CW Tau. This suggests that $\mathrm{CW}$ Tau may be a binary with only one component, CW Tau/e, undergoing collimated mass outflow. In fact, the morphology of CW Tau can be very well reproduced by co-adding two pointlike sources with similar luminosities, the sources being aligned in the east-west direction and separated 0.2 (28 AU). The residuals of the fitting are $\sim 4 \%$ of the total flux. There are also independent indications that CW Tau may be a binary (BSCG).

If the two components have similar masses, the Lagrangian point of the system will be at the middle point between the two stars, indicating a characteristic size of $\sim 14 \mathrm{AU}$ for the accretion disk around any of the components. Therefore, if the millimeter flux from the TTSs is mainly produced in large-scale (100 AU) accretion disks, CW Tau could be significantly

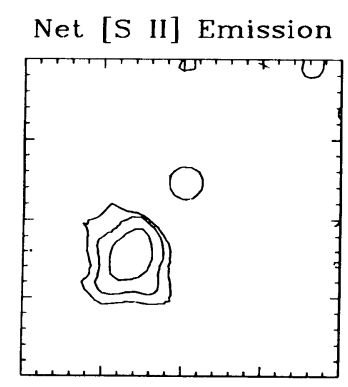

Net $[\mathrm{N}$ II $]+\mathrm{H} \alpha$ Emission

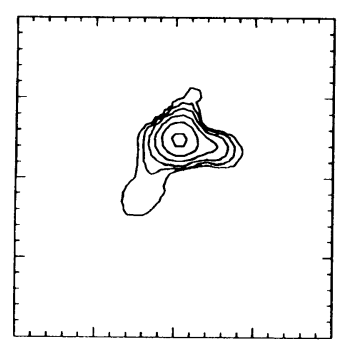

Fig. 3.-Net [ $\left[\begin{array}{ll}\mathrm{S} & \mathrm{II}\end{array}\right]$ (top) and [ $\mathrm{N}$ II] $+\mathrm{H} \alpha$ (bottom) line emission. The isocontour levels and the scale are the same as in Figs. 1 and 2. 
fainter than other TTSs at these wavelengths. In fact, the mass of CW Tau's disk is $0.0013 M_{\odot}$ (Beckwith \& Sargent 1991), an order of magnitude smaller than other TTSs with similar IR luminosities and forbidden line strengths (e.g., DO Tau or DR Tau).

$\mathrm{CW}$ Tau has been classified as $\mathrm{K} 3(\mathrm{CK})$ with mass $M_{*}=$ $1.40 M_{\odot}$ and a luminosity $L_{*}=2.6 L_{\odot}$. If the two components of $\mathrm{CW}$ Tau are assumed to have similar luminosities and masses, the inferred orbital period is $88 \mathrm{yr}$. Spectral variations have been reported in a time scale of $11 \mathrm{yr}$ (see Rydgren, Strom, \& Strom 1976; Walker 1987) that could be related to the possible binarity of CW Tau.

\subsection{Constraints on Models for FLER Formation}

There are two basic models for FLER formation. HL proposed that FLERs are formed at distances of 50-100 AU in the regions where the TTS winds shock against the flaring circumstellar disks and heat. A different possibility is that FLERs are formed in magnetically pinched outflows ejected centrifugally from the inner parts of the accretion disk (GP). The temperature and density attained by the outflow in the mild shocks at the pinched areas (also called focal points) readily account for FLERs (details in GP). Jets can be initiated at the focal points. CW Tau observations show that the FLER was an unresolved jet (size $\simeq 200 \mathrm{AU}$ ). Moreover, the jet is visible from a distance of $176 \mathrm{AU}$ from the star, indicating that the mechanism generating the outflow has to be able to heat the gas, at this long distance. Hydromagnetic winds from Keplerian accretion disks can recollimate, forming focal regions (Blandford \& Payne 1982; Pelletier \& Pudritz 1992) where mild MHD shocks heat the gas provided that they achieve high enough (fast magnetosonic) Mach number $n \simeq\left[(4 \pi \rho)^{1 / 2} V_{\infty} / B\right]$ (where $\rho$ is the gas density, $B$ the magnetic field strength, and $V_{\infty}$ the terminal speed of the wind). The focal distance can be found in terms of the maximum radius of the flow $(\mathrm{GP} ; \mathrm{PP})$ :

$$
\frac{Z_{\text {foc }}}{r_{\max }}=2\left[3\left(n^{2}+1\right)\right]^{1 / 2}
$$

this radius is very sensitive to the accretion rate. Taking $r_{\max } \simeq$ 14 AU and $n \simeq 2.4$ for the critical Mach number for which flows begin to recollimate, we find $z_{\text {foc }} \simeq 126 \mathrm{AU}$, in reasonable agreement with the observed 176 AU (see GP for details). However, for the typical accretion rates found in the TTS, disk winds are expected to depart only from the inner $2 \mathrm{AU}$ of the accretion disk (GP), and in this case $n$ has to be $\simeq 25$ so equation (1) is satisfied. This large Mach number implies that the flow is mainly poloidal and, therefore, has a much larger velocity component along the outflow axis than in the toroidal direction (rotation around the disk axis).

In conclusion, the observations resolve CW Tau's FLER into a jet already collimated at $176 \mathrm{AU}$ from the star that is visible in [S II] up to $300 \mathrm{AU}$ from the star $\left(\sim 2^{\prime \prime}\right)$. CW Tau is extended in the direction P.A. $\sim 0^{\circ}$ with an aspect ratio of $2: 1$. This extension suggests that $\mathrm{CW}$ Tau is a binary system with two components separated $28 \mathrm{AU}$. The data are consistent with the hypothesis that FLERs are shocks at the focal points in recollimating, hydromagnetic disk winds.

Ralph E. Pudritz has been my collaborator in these ventures, and I thank him for many long and fruitful discussions about accretion disks and magnetic fields. I am indebted to Marshall McCall, who lent us his interference filters for these observations. I also thank our resident astronomer Jerome Bouvier and the staff at CFHT for their support during the observations, and very especially Robert McClure for his very helpful comments on CFHT + HRC optical properties. I acknowledge NSERC of Canada and the Ministerio de Educación y Ciencia of Spain for the postdoctoral fellowships that have allowed me to carry out this work.

\section{REFERENCES}

Beckwith, S. V., Sargent, A. I., Chini, R. S., \& Güsten, R. 1990, AJ, 99, 924 (BSCG)

Beckwith, S. V., \& Sargent, A. I. 1991, ApJ, 380, 250

Benson, P. J., \& Myers, P. C. 1989, ApJS, 71, 89

Blandford, R. D., \& Payne, D. R. 1982, MNRAS, 199, 883

Cabrit, S., Edwards, S., Strom, S. E., \& Strom, K. M. 1990, ApJ, 354, 687

Cohen, M., \& Kuhi, L. V. 1979, ApJS, 41, 743 (CK)

Edwards, S. Cabrit, S., Strom, S. E., Heyer, I., Strom, K. M., \& Anderson, E. 1987, ApJ, 321, 473

Gómez de Castro, A. I., \& Pudritz, R. E. 1992, ApJ, 397, L107

. 1993, ApJ, 409, 748 (GP)

Hartmann, L., \& Raymond, J. C. 1988, ApJ, 337, 903 (HL)
Hirth, R., Mundt, R., \& Solf, J. 1993, in preparation

Lucy, L. B. 1974, AJ, 79, 745

McClure, R. D., Grundmann, W. A., Rambold, W. N., Fletcher, J. M., Richardson, E. H. \& Stilburn, J. R. 1989, PASP, 101, 1156

McClure, R. D., Arnaud, J., Fletcher, J. M., Nieto, J. L., \& Racine, R. 1991, PASP, 103, 570

Pelletier, G., \& Pudritz, R. E. 1992, ApJ, 394, 117

Rydgren, A. E., Strom, S. E.. \& Strom, K. M. 1976, ApJS, 30, 307

Scheffer, H. 1982 in Landolt-Börnstein, New Ser., Group VI, Vol. 2, Subvol. c, Interstellar Matter-Galaxy-Universe, ed. K. Schaifers \& H. H. Voigt (Berlin: Springer-Verlag), 47

Walker, M. F. 1987, PASP, 99, 392 